\title{
Complex Model of Process of Investigation of Guided Missiles with Optical Heads of Homing
}

\author{
Alexander N. Glushkov, \\ Yurii L. Koziratsky and Ruslan E. Merkulov* \\ Military Education and Research Centre of Military-Air Forces \\ «Military-Air Academy \\ Named After Professor N.E. Zhukovsky and Yu.A. Gagarin» \\ 54a Starykh Bolshevikov Str., Voronezh, 394064, Russia
}

Received 03.08.2016, received in revised form 10.02.2017, accepted 02.01.2018

Results of working out of complex mathematical model of process of investigation of guided missiles with optical heads of homing are presented. Unlike known models the modules reflecting subprocesses by means of a locator to observe of supervision and detection of guided missiles with optical heads of homing, and also works of a subsystem of investigation fixing to time balance are in addition included in the given model.

Keywords: Homing missiles, laser, functional model.

Citation: Glushkov A.N., Koziratsky Yu.L., Merkulov R.E. Complex model of process of investigation of guided missiles with optical heads of homing, J. Sib. Fed. Univ. Eng. technol., 2018, 11(1), 29-36. DOI: 10.17516/1999-494X-0004.

\section{Комплексная модель \\ процесса разведки управляемых ракет \\ с оптическими головками самонаведения}

\author{
А.Н. Глушков, Ю.Л. Козирацкий, Р.Е. Меркулов \\ Военный учебно-научный центр Военно-воздушных сил \\ «Военно-воздушная академия \\ имени профессора Н.Е. Жуковского и Ю.А. Гагарина» \\ Россия, 394064, Воронеж, ул. Старых Большевиков, 54 а
}

Представлены результаты разработки комплексной математической модели процесса разведки управляемых ракет с оптическими головками самонаведения. В отличие от известных моделей в данную модель дополнительно включены модули, отражаюшие подпроцессы локационного наблюдения и обнаружения управляемых ракет с оптическими

(c) Siberian Federal University. All rights reserved

* Corresponding author E-mail address: rus_212@mail.ru, urleo@bk.ru 
головками самонаведения, а также фиксирующие временной баланс работы подсистемы разведки.

Ключевые слова: головка самонаведения ракеты, лазерное излучение, функциональная модель.

\section{Введение}

Современный этап развития средств вооруженной борьбы характеризуется широким использованием управляемых ракет (УР) с оптическими головками самонаведения (ОГС) для уничтожения летательных аппаратов (ЛА) противника. Это обуславливает необходимость создания бортовых комплексов обороны (БКО) ЛА, предназначенных для их защиты от УР. БКО включает в себя подсистему разведки (ПСР), подсистему создания помех (ПСП) и подсистему управления [1]. При проектировании и исследовании сложных технических систем, к которым обычно относятся системы вооружений, широко используют их моделирование [2]. В настоящей работе приводятся результаты разработки комплексной математической модели процесса разведки УР с ОГС с целью определения оптимальных структуры и значений параметров ПСР лазерного БКО ЛА.

\section{Постановка задачи}

Структурная схема модели ПСР представлена на рис. 1.

В общей структуре процесса разведки атакующих ЛА УР модель отражает подпроцессы поиска и обнаружения УР, определение типа ее головки самонаведения (оптическая/радиолокационная), измерение текущих координат, а также временной баланс работы ПСР. В отличие от известных моделей, формализующих вероятность успешного завершения поиска объекта, в нее дополнительно включены последовательно соединенные с модулем поиска модули, отражающие подпроцессы локационного наблюдения и обнаружения УР с ОГС. Модуль поиска позволяет проводить исследование эффективности поиска атакующей УР для двух вариантов построения ПСР (в обоих вариантах она состоит из пассивного и активного каналов). В первом варианте рассматривается ПСР на основе работающего в ИК- или УФ-области спектра пеленгатора и лазерного локатора. Пеленгатор осуществляет поиск и обнаружение атакующей УР по излучению факела ее двигателя, а с помощью лазерного локатора на основе бликовой локации производится определение типа ГСН УР, тем самым реализуется процедура двухэтапного просмотра заданной области пространства [3]. Во втором варианте построения ПСР рассматрива-

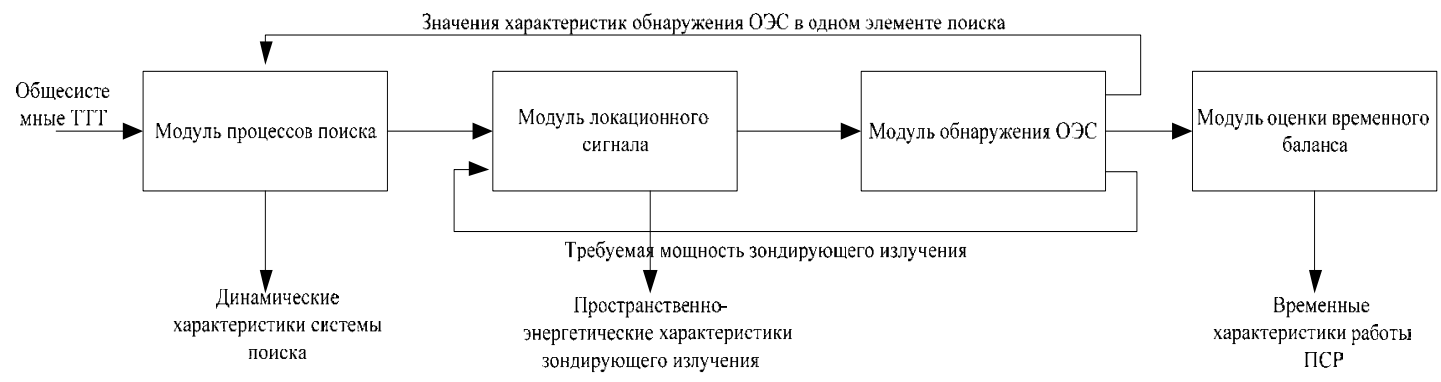

Рис. 1. Структурная схема комплексной модели процесса разведки УР с ОГС

Fig. 1. The Block diagramme of complex model of process of investigation Ur with OGS 
ется случай, когда в состав подсистемы включены УФ- и ИК-пеленгаторы, а также лазерный локатор [4]. Принятие решения об обнаружении атакующей УР может производиться с использованием логики как «И», так и «ИЛИ». Определение типа ГСН УР осуществляется по аналогии с первым вариантом построения ПСР.

\section{Решение}

В качестве показателей эффективности выполнения поиска формализованы финальная вероятность успешного завершения поиска $P_{O D}$, вероятность успешного завершения поиска за заданное время $\mathrm{P}_{D}(t)$, а также среднее время поиска $\mathrm{m}_{\mathrm{D}}$. Данные показатели определяются следующими выражениями [3, 4]:

Первый вариант построения ПСР

$$
\begin{gathered}
P_{O D}=\sum_{i=1}^{m} \sum_{k=1}^{n} \sum_{u=0}^{\infty} \sum_{v=0}^{\infty} P_{1 i} P_{2 k} D_{1 i} D_{2 k} \times \prod_{j=1}^{i-1}\left(1-F_{1 j}\right) \times \prod_{\ell=1}^{k-1}\left(1-F_{2 k}\right) \cdot a_{0}^{u} b_{1}^{v} ; \\
P_{D}(t)=\sum_{i=1}^{m} \sum_{k=1}^{n} \sum_{u=0}^{\infty} \sum_{v=0}^{\infty} P_{1 i} P_{2 k} D_{1 i} D_{2 k} \times \prod_{j=1}^{i-1}\left(1-F_{1 j}\right) \prod_{\ell=1}^{k-1}\left(1-F_{2 k}\right) a_{0}^{u} b_{1}^{v} h\left\{t-(m u+i) \tau_{1}-(n v+k) \tau_{2}\right\}, \\
m_{D}=\sum_{i=1}^{m} \sum_{k=1}^{n} \sum_{u=0}^{\infty} \sum_{v=0}^{\infty} P_{1 i} P_{2 k} D_{1 i} D_{2 k} \times \prod_{j=1}^{i-1}\left(1-F_{1 j}\right) \prod_{\ell=1}^{k-1}\left(1-F_{2 \ell}\right) a_{0}^{u} b_{1}^{v}\left[(m u+i) \tau_{1}+(n v+k) \tau_{2}\right],
\end{gathered}
$$

где $a_{0}=\sum_{i=1}^{m} P_{1 i}\left(1-D_{1 i}\right) \prod_{j=1}^{m}\left(1-F_{1 j}\right) ; \quad b_{1}=\sum_{k=1}^{n} P_{2 k}\left(1-D_{2 k}\right) \prod_{\substack{\ell=1 \\ \ell \neq k}}^{n}\left(1-F_{2 \ell}\right) ; P_{i}, P_{2 k}-$ априорная вероятность наличия цели в $i$-том и $k$-том элементах разрешения секторов поиска первого и второго средства, осуществляющего поиск и обнаружение, соответственно; $D_{1 i}, F_{1 i}, D_{2 k}, F_{2 k}$ - вероятности правильного обнаружения и ложной тревоги при обнаружении цели в і-том и k-том элементах разрешения секторов поиска; $m, n$ - количество элементов разрешения в секторах поиска пассивного и активного каналов; $h(\cdot)$ - единичная функция; $\tau_{1}, \tau_{2}$ - время просмотра одного элемента разрешения пассивным и активным каналом.

\section{Второй вариант построения ПСР}

\section{А. Решение об обнаружении цели принимается}

в соответствии с логикой «ИЛИ»

В данном случае вероятность $P_{D}(t)$ и финальная вероятность $P_{O D}$ определяются как вероятности выполнения хотя бы одного из двух событий: «произошло обнаружение цели в первом (втором) канале», т.е.

$$
\begin{gathered}
P_{O D}=P_{O D 1}+P_{O D 2}-P_{O D 1} \cdot P_{O D 2}, \\
P_{D}(t)=P_{D 1}(t)+P_{D 2}(t)-P_{D 1}(t) \cdot P_{D 2}(t),
\end{gathered}
$$

где $P_{O D 1}, P_{O D 2}-$ финальные вероятности обнаружения цели пассивным и активным каналами соответственно; $\mathrm{P}_{D 1}(t), \mathrm{P}_{D 2}(t)$ - вероятности обнаружения цели к моменту времени $\mathrm{t}$ соответственно пассивным и активным каналами.

Математическое ожидание времени успешного завершения поиска определяется выражением 


$$
m_{D}=\frac{1}{P_{O D}} \int_{0}^{\infty} t P_{D}(t) d t
$$

Вероятности $\mathrm{P}_{D 1}(t), \mathrm{P}_{D 2}(t), P_{O D 1}, P_{O D 2}$ определяются выражениями, аналогичными (1):

$$
\begin{gathered}
P_{D 1}(t)=\sum_{i=1}^{m} \sum_{k=1}^{n} \sum_{u=0}^{\infty} \sum_{v=0}^{\infty} P_{1 i} P_{2 k} D_{1 i} D_{2 k} \prod_{j=1}^{i-1}\left(1-F_{1 j}\right) \prod_{\ell=1}^{k-1}\left(1-F_{2 \ell}\right) a_{0}^{u} \cdot b_{1 I}^{v} h\left\{t-(m u+i) \tau_{1}-(n v+k) \tau_{2}\right\}, \\
P_{D 2}(t)=\sum_{i=1}^{m} \sum_{j=1}^{\ell} \sum_{u=0}^{\infty} \sum_{w=0}^{\infty} P_{1 i} P_{3 j} D_{1 i} D_{3 j} \prod_{\Theta=1}^{i-1}\left(1-F_{1 \Theta}\right) \prod_{\varepsilon=1}^{j-1}\left(1-F_{3 \varepsilon}\right) a_{0}^{u} \cdot b_{1 I I}^{w} h\left\{t-(m u+i) \tau_{1}-(\ell w+j) \tau_{3}\right\}, \\
P_{O D 1}=\left.P_{D 1}(t)\right|_{h(x)=1} ; \quad P_{O D 2}=\left.P_{D 2}(t)\right|_{h(x)=1},
\end{gathered}
$$

где $a_{0}=\sum_{i=1}^{m} P_{1 i}\left(1-D_{1 i}\right) \prod_{\substack{j=1 \\ i \neq i}}^{m}\left(1-F_{1 j}\right) ; \quad b_{1 I}=\sum_{k=1}^{n} P_{2 k}\left(1-D_{2 k}\right) \prod_{\substack{\ell=1 \\ \ell \neq i}}^{n}\left(1-F_{2 \ell}\right) ; b_{1 I}=\sum_{j=1}^{\ell} P_{3 j}\left(1-D_{3 j}\right) \prod_{\substack{\Theta-1 \\ \Theta \neq j}}^{n}\left(1-F_{3 \Theta}\right) ; 1-$ число элементов разрешения в зоне поиска лазерного локатора. Индексы 1, 2, 3 соответствуют характеристикам ИК- и УФ-пеленгаторов, а также лазерного локатора.

Выражение для математического ожидания времени успешного завершения поиска имеет следующий вид:

$$
m_{D}=\frac{1}{P_{0 D}}\left(\frac{D_{1} D_{2}}{m \cdot n} a_{3}\left(1-b_{4}\right)+\frac{D_{1} D_{3}}{m \cdot l} b_{3}\left(1-a_{4}\right)\right)
$$

где $\mathrm{a}_{3}=\sum_{\mathrm{i}=1}^{\mathrm{m}} \sum_{\mathrm{k}=1}^{\mathrm{n}} \sum_{\mathrm{u}=0}^{\infty} \sum_{\mathrm{v}=0}^{\infty}\left(1-\mathrm{D}_{1}\right)^{\mathrm{u}}\left(1-\mathrm{F}_{1}\right)^{(\mathrm{m}-1) \mathrm{u}+\mathrm{i}-1} \cdot\left(1-\mathrm{D}_{2}\right)^{\mathrm{v}}\left(1-\mathrm{F}_{2}\right)^{(\mathrm{n}-1) \mathrm{v}+\mathrm{k}-1}\left((\mathrm{mu}+\mathrm{i}) \tau_{1}-(\mathrm{nv}+\mathrm{k}) \tau_{2}\right)$;

$\mathrm{b}_{3}=\sum_{\mathrm{i}=1}^{\mathrm{m}} \sum_{\mathrm{j}=1}^{1} \sum_{\mathrm{u}=0}^{\infty} \sum_{\mathrm{w}=0}^{\infty}\left(1-\mathrm{D}_{1}\right)^{\mathrm{u}}\left(1-\mathrm{F}_{1}\right)^{(\mathrm{m}-1) \mathrm{u}+\mathrm{i}-1} \cdot\left(1-\mathrm{D}_{3}\right)^{\mathrm{w}}\left(1-\mathrm{F}_{3}\right)^{(1-1) \mathrm{w}+\mathrm{j}-1}\left((\mathrm{mu}+\mathrm{i}) \tau_{1}-(\mathrm{lw}+\mathrm{j}) \tau_{3}\right)$;

$a_{4}=\sum_{i=1}^{m} \sum_{k=1}^{n} \sum_{u=0}^{\infty} \sum_{v=0}^{\infty}\left(1-D_{1}\right)^{u}\left(1-F_{1}\right)^{(m-1) u+i-1} \cdot\left(1-D_{2}\right)^{v}\left(1-F_{2}\right)^{(n-1) v+k-1}$;

$b_{4}=\sum_{i=1}^{m} \sum_{j=1}^{l} \sum_{u=0}^{\infty} \sum_{w=0}^{\infty}\left(1-D_{1}\right)^{u}\left(1-F_{1}\right)^{(m-1) u+i-1} \cdot\left(1-D_{3}\right)^{w}\left(1-F_{3}\right)^{(l-1) w+j-1}$.

\section{Б. Решение об обнаружении цеели принимается}

в соответствии с логикой «И»

Когда решение об обнаружении цели принимается в соответствии с логикой «и», вероятности $\mathrm{P}_{\mathrm{OD}}, \mathrm{P}_{\mathrm{D}}(\mathrm{t})$ определяются следующим образом:

$$
\begin{gathered}
P_{O D}=P_{O D 1} \cdot P_{O D 2} ; \\
P_{D}(t)=P_{D 1}(t) \cdot P_{D 2}(t),
\end{gathered}
$$

где $\mathrm{P}_{D 1}(t), P_{O D 2}, \mathrm{P}_{D 1}(t), P_{O D 2}$ определяются выражениями (4).

Математическое ожидание времени успешного завершения поиска может быть найдено по формуле

$$
m_{D}=\frac{1}{P_{0 D}}\left(\frac{D_{1} D_{2}}{m \cdot n} a_{3} b_{4}+\frac{D_{1} D_{3}}{m \cdot l} b_{3} a_{4}\right)
$$




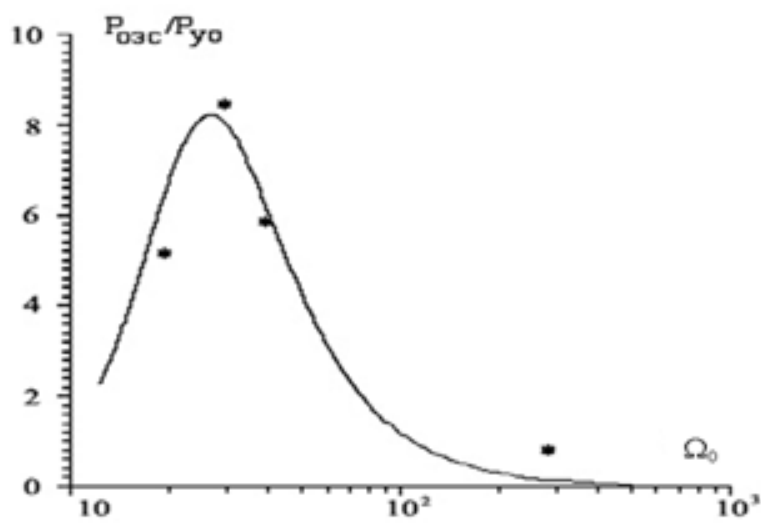

Рис. 2. Зависимость отношения $P_{\text {оэс }} / P_{y o}$ от числа Френеля $\Omega_{0}$

Fig. 2. Dependence of the relation $P_{о э с} / P_{y o}$ on number of Frenelja $\Omega_{0}$

Расчет характеристик обнаружения атакующей ракеты ИК- и УФ-пеленгаторами производится на основе модели излучения факела двигателя УР [5] с использованием методики [6]. Энергетическое ослабление излучения факела ракеты атмосферой может быть учтено известными методами [7]. Ниже остановимся на задаче обнаружения ОГС УР, решаемой в модулях локационного наблюдения и обнаружения ОГС разработанной модели.

В модуле локационного наблюдения ОГС производится описание процесса трансформации функции когерентности зондирующего излучения локатора при его распространении в турбулентной атмосфере от излучающей апертуры локатора к ОГС и обратно к фотоприемнику локатора. Упомянутая функция когерентности формализована для ОГС с матричным фотоприемником смотрящего типа [8]. На рис. 2 отражены результаты экспериментальной проверки данной модели. Сплошной кривой приведена зависимость от числа Френеля отношения средних мощностей локационного сигнала отраженного от ПНВ и уголкового отражателя, полученная с использованием разработанной модели. Знаком «*» обозначены измеренные значения этого отношения. Данные результаты подтверждают возможность использования разработанной модели для описания локационного сигнала.

В модуле обнаружения ОГС формализован процесс принятия решения по достаточной статистике:

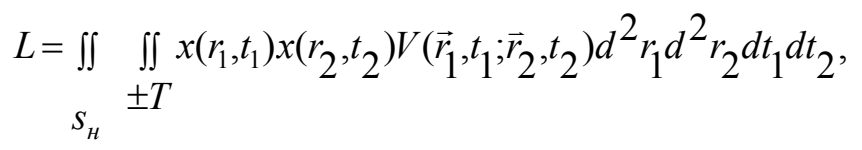

формируемой на выходе приемника локатора по наблюдаемой аддитивной смеси реализации отраженного от ОГС поля и фона. Передаточная функция приемного устройства $V(\square)$ выявлена в результате решения интегрального уравнения Фредгольма второго рода, найденного с использованием разложения Карунена-Лоэва [9]:

$$
\begin{gathered}
V\left(x_{1}, x_{2}\right)=\frac{P_{c}}{N_{0}^{2}} \sum_{n=0}^{\infty} \frac{\varphi_{n}\left(x_{1}\right) \varphi_{n}\left(x_{2}\right)}{\chi_{n}\left[1+\frac{P_{c}}{N_{0} \chi_{n}}\right]}, \\
-33-
\end{gathered}
$$


где $x_{1}=t_{1} / T, x_{2}=t_{2} / T ; P_{c}, N_{0}$ - мощность локационного сигнала и спектральная плотность фона соответственно; $\varphi_{n}(x)$ - собственные функции; $\left\{\chi_{n}\right\}$ - соответствующая система собственных значений интегрального уравнения

$$
\varphi(x)=\chi \int_{ \pm 1} R_{c}\left(x, x_{1}\right) \varphi\left(x_{1}\right) d x_{1}
$$

и введения пробной системы ортонормированных функций, определенной на интервале [-1...1] и удовлетворяющей следующим условиям [10]:

$$
\begin{gathered}
\int_{ \pm 1} \psi_{n}\left(x_{1}\right) \psi_{k}\left(x_{2}\right) \gamma_{c}\left(x_{1}, x_{2}\right) d x_{1} d x_{2}=\delta_{n, k} / \tilde{\chi}_{n} \int_{ \pm 1} \psi_{n}(x) \psi_{k}(x) d x=\delta_{n, k} \\
\int_{ \pm 1} \psi_{n}(x) \psi_{k}(x) d x=\delta_{n, k}
\end{gathered}
$$

где $\gamma_{c}\left(x_{1}, x_{2}\right)$ - положительно определенная функция, непрерывно симметрическая по обоим переменным; $R_{c}(\square)$ - временная корреляционная функция отраженного сигнала.

Для описания влияния динамических параметров локатора на эффективность разведки ОГС учтена связь между модулями поиска и обнаружения ОГС, которая порождена функциональной зависимостью показателей успешного завершения поиска от характеристик обнаружения ОГС. Для описания влияния характеристик приемопередающего устройства локатора на эффективность разведки учтена связь между подпроцессами обнаружения и локационного наблюдения ОГС, которая порождена функциональной зависимостью характеристик обнаружения от параметров оптических схем объектов разведки и параметров приемопередающего тракта локатора.

\section{Заключение}

Таким образом, разработанная модель подсистемы разведки позволяет комплексно исследовать показатели эффективности разведки УР с ОГС в зависимости от параметров их оптических схем и параметров ПСР, а также условий распространения излучения. Это открывает возможность оптимизации значений параметров подсистем разведки БКО ЛА.

\section{Список литературы}

[1] Бутузов В.А. Лазер на защите воздушных судов. Военный парад: сетевой журнал. 2011. URL: http://www. Milparade.com [Butuzov V.A. The laser on protection of aircrafts. Military parade: Network magazine. 2011. URL: http://www. Milparade.com (in Russian)].

[2] Гайкович А.И. Основы теории проектирования сложных технических систем. СПб., НИЦ «МОРИНТЕХ», 2001. 432 c. [Gaikovihc A.I. Bas of the theory of designing of difficult technical systems. SPb.: FACE DOWNWARDS «MORINTEX», 2001. 432 p. (in Russian)].

[3] Глушков А.Н., Козирацкий Ю.Л. Лысиков В.Ф. Математическая модель двух этапного поиска случайно появляющихся целей. Радиотехника. 1999, 40 (6). 4-9. [Glushkov A.N., Koziratsky Ju.L. Lysikov V.F. Mathematical model of two gradual search of casually appearing purposes. Radio engineering. 1999, 40 (6). 4-9 (in Russian)].

[4] Глушков А.Н., Козирацкий Ю.Л., Лысиков В.Ф. Показатели эффективности комплексированной системы разведки на этапе поиска и обнаружения. Радиотехника. 2000 (8), 12-16. [Glushkov A.N., Koziratsky Ju.L., Lysikov V.F. Pokazateli of efficiency the integrated

$$
-34-
$$


investigation systems at a search and detection stage. Radio engineering. 2000, (8), 12-16 (in Russian)].

[5] Алексеев О.А. Математическая модель спектральной плотности силы излучения факелов, образующихся при стационарном режиме горения топлив. Оптический журнал. 1998, 65, (1), 51-54. [Alekseev O.A. Mathematical model of spectral density of force of radiation of the torches formed at a stationary mode of burning топлив. Optical magazine. 1998, 65 (1), 51-54 (in Russian)].

[6] Поветко В.Н., Понькин В. А. и др. Критерии, методы и математические модели оценки оптической заметности объектов ВВТ: научно-методические материаль. М., Воениздат, 1990 [Povetko V.N, Ponkin V.A, etc. Criterion, methods and mathematical models of an estimation of an optical visibility of objects VVT: scientifically-methodical materials. Moscow, Military publishing house, 1990 (in Russian)].

[7] Зуев В.Е. Распространение лазерного излучения в атмосфере. М., Радио и связь, 1981. [Zuev V.E. Distribution of laser radiation to atmosphere. M, Radio and communication, 1981 (in Russian)].

[8] Глушков А.Н., Кравцов Р.Н., Митрофанов А.Л. Модель локационного наблюдения ОЭС, Информационно-измерительные и управляющие системы. 2006 (7), 23-29. [Glushkov A.N., Kravtsov R.N, Mitrofanov A.L. Model локационного supervision ОЭС, Information-measuring and operating systems. 2006 (7), 23-29(in Russian)].

[9] Глушков А.Н., Кравцов Р.Н., Митрофанов А.Л Об одном подходе к численному решению интегральных уравнений Фредгольма в задачах синтеза информационных систем, Теория и техника радиосвязи. 2005 (2), 7-15. [Glushkov A.N., Kravtsov R.N, Mitrofanov A.L. About one approach to the numerical decision of the integrated equations of Fredgolma in problems of synthesis of information systems, The Theory and technics of a radio communication. 2005 (2), 7-15 (in Russian)].

[10] Глушков А.Н., Кравцов Р.Н., Митрофанов А.Л. Численный синтез и анализ оптимального обнаружителя, Теория и техника радиосвязи. 2004 (1), 84-96. [Glushkov A.N., Kravtsov R.N., Mitrofanov A.L. Numerical synthesis and the analysis optimum detector, The Theory and technics of a radio communication. 2004 (1), 84-96 (in Russian)].

[11] Козирацкий Ю.Л. Определение оптимального числа зондирующих посылок лазернойлокационной системы в условиях атмосферных аэрозольных помех, Журнал «Информационный конфликт в спектре электромагнитных волн», 1994 (1) (Приложение к журналу «Радиотехника»), 90-95. [Koziratsky Ju.L. Definition of optimum number of probing parcels of laser-lokatsionnoj system in the conditions of atmospheric aerosol hindrances, Magazine "the Information conflict in a spectrum of electromagnetic waves", 1994 (1) (the Appendix to magazine "Radio engineering"), 90-95 (in Russian)].

[12] Козирацкий Ю.Л., Аниканов А.В. Поиск целей оптико-электронными системами среди контрастных выбросов, Информационный конфликт в спектре электромагнитных волн, 1994, (1) (Приложение к журналу «Радиотехника»), 95-98. [Koziratskiy Ju.L., Anikanov A.V. Search of the purposes optiko-electronic systems among contrast emissions, Magazine "the Information conflict in a spectrum of electromagnetic waves", 1994 (1) (the Appendix to magazine "Radio engineering"), 95-98 (in Russian)]. 
[13] Козирацкий Ю.Л. Поиск цели оптико-электронными системами в условиях нестационарных атмосферных аэрозольных помех, Информаиионный конфликт в спектре электромагнитных волн, 1994, (1) (Приложение к журналу «Радиотехника») 88-89. [Koziratsky Ju.L. Search of the purpose optiko-electronic systems in the conditions of non-stationary atmospheric aerosol hindrances, Magazine "the Information conflict in a spectrum of electromagnetic waves", 1994 (1) (the Appendix to magazine "Radio engineering") 88-89 (in Russian)].

[14] Козирацкий Ю.Л. и др. Модели информационного конфликта средств поиска и обнаружения. М., Радиотехника, 2013, 232. [Koziratskiy Ju.L., etc. Models of the information conflict of means of search and detection. M: Radio engineering, 2013, 232 (in Russian)].

[15] Козирацкий Ю.Л. и др. Модели пространственного и частотного поиска. М., Paдиотехника, 2014, 342. [Koziratskij Ju.L., etc. Models of spatial and frequency search. M: Radio engineering, 2014, 342 (in Russian)].

[16] Козирацкий Ю.Л. и др. Обнаружение и координатометрия оптико-электронных средств, оценка параметров их сигналов. М., Радиотехника, 2015, C. 454. [Koziratskiy Ju.L., etc. Detection and coordinate metrology optiko-electronic means, an estimation of parametres of their signals. M., Radio engineering, 2015, 454 (in Russian)]. 